\title{
Liste de contrôle pour laboratoires d'intervention contre les éclosions de maladie infectieuse - Considérations relatives aux mesures de préparation et interventions face aux menaces émergentes
}

\author{
Tracie EisBrenner ${ }^{1 *}$, Graham Tipples ${ }^{2,3}$, Theodore Kuschak ${ }^{1,3,4}$, Matthew Gilmour ${ }^{1,3,4}$
}

\section{Résumé}

L'objectif de la Liste de contrôle pour laboratoires d'intervention contre les éclosions de maladie infectieuse (la «liste de contrôle») est de fournir aux laboratoires de santé publique et aux réseaux de laboratoires opérant à plusieurs niveaux de juridiction un outil utile et adaptable afin d'aider à identifier rapidement les considérations importantes relatives aux interventions en cas d'éclosions, notamment lors d'une étude visant une menace de maladie infectieuse jusqu'alors inconnue. La liste de contrôle a été élaborée par le Laboratoire national de microbiologie du Canada en collaboration avec des experts de laboratoires provinciaux, territoriaux, nationaux et internationaux, dont le Réseau des laboratoires de santé publique du Canada et le Réseau des laboratoires du Groupe de travail sur la protection de la santé mondiale. Bien que la liste de contrôle ait été initialement conçue pour refléter les leçons tirées de la participation des laboratoires nationaux de microbiologie à des interventions étendues à des épidémies nationales et internationales (e.g. l'épidémie du virus Zika [2015 à 2016] et l'épidémie du virus Ebola, en Afrique de l'Ouest [2014 à 2016]), l'importance d'optimiser la coordination des interventions des laboratoires n'a été soulignée que par les défis permanents présentés par les exigences des interventions à la pandémie de COVID-19. La liste de contrôle identifie cinq thèmes d'intervention en laboratoire hautement interdépendants, dont chacun englobe de multiples considérations qui peuvent être essentielles à une intervention coordonnée et stratégique en cas d'épidémie. En tant que tel, l'examen complet des considérations de la liste de contrôle par les organisations de laboratoires intervenants peut fournir une occasion importante de détecter rapidement les principales considérations et interdépendances en matière d'intervention, et d'atténuer les risques susceptibles d'avoir une incidence sur l'action de santé publique.
Cette oeuvre est mise à la disposition selon les termes de la licence internationale Creative Commons Attribution 4.0

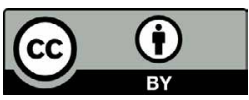

Affiliations

${ }^{1}$ Laboratoire national de microbiologie, Winnipeg, MB

${ }^{2}$ Laboratoire provincial de santé publique de l'Alberta, Alberta Precision Laboratories, Edmonton, $A B$

${ }^{3}$ Réseau des laboratoires de santé publique du Canada

${ }^{4}$ Réseau de laboratoires du Groupe de travail sur la protection de la santé mondial

*Correspondance :

tracie.eisbrenner@canada.ca

Citation proposée : EisBrenner T, Tipples G, Kuschak T, Gilmour M. Liste de contrôle pour laboratoires

d'intervention contre les éclosions de maladie infectieuse - Considérations relatives aux mesures et interventions face aux menaces émergentes. Relevé des maladies transmissibles au Canada 2020;46(10):351-63.

https://doi.org/10.14745/ccdr.v46i10a01f

Mots-clés : liste de contrôle, COVID-19, Ebola, maladie infectieuse émergente, gestion des urgences, agents pathogènes à haute conséquence, maladie infectieuse, intervention des laboratoires, préparation aux épidémies, interventions contre les épidémies, laboratoire de santé publique, SRAS, SRAS-CoV-2, Zika

\section{Introduction}

Les interventions contre les épidémies de maladies infectieuses posent des défis et des considérations uniques aux laboratoires, en particulier lorsqu'ils étudient une maladie infectieuse auparavant inconnue ou nouvellement définie (1). Grâce à la participation importante du Laboratoire national de microbiologie aux interventions nationales et internationales contre les épidémies et les pandémies, un certain nombre de considérations et d'enseignements clés ont été mis en 
évidence. Il est notamment nécessaire que les laboratoires $d$ 'intervention 1) élaborent et déploient rapidement de nouvelles méthodes de test diagnostique spécifiques aux nouveaux agents pathogènes, 2) participent à l'élaboration collaborative et itérative de définitions de cas et de critères de test pour tenir compte de l'évolution des preuves scientifiques à mesure que l'épidémie progresse, 3) engagent stratégiquement les partenaires de la santé publique pour optimiser la capacité de réaction et la coordination, et 4) établissent des processus et des procédures de partage d'information qui permettent aux laboratoires de santé publique d'étudier, de surveiller, d'effectuer des recherches, pour transmettre des messages de santé publique et d'agir en temps utile (2). L'évolution constante de la génomique de la santé publique et des autres approches "omiques » présente des défis supplémentaires et offre d'importantes possibilités d'améliorer la capacité d'intervention contre les maladies infectieuses $(3,4)$. Étant donné la nature complexe et spécifique des considérations relatives aux interventions des laboratoires, une coordination efficace et en temps voulu peut s'avérer difficile en absence d'une approche stratégique et structurée.

L'utilité potentielle d'une approche par liste de contrôle pour renforcer la préparation des laboratoires et la coordination des interventions a été récemment soulignée lors de l'engagement prolongé du Laboratoire national de microbiologie dans les efforts d'intervention nationaux et internationaux, en particulier durant l'épidémie du virus Ebola en Afrique occidentale (2014 à 2016) et l'épidémie du virus Zika (2015 à 2016) (5-8). Dans ce contexte, l'élaboration d'une liste de contrôle a également été envisagée en fonction des résultats souhaités énoncés dans le Plan stratégique 2016-2020 du Réseau des laboratoires de santé publique du Canada y compris la «Priorité 2 : Renforcer la capacité d'intervention coordonnée pour faire face aux agents pathogènes établis, émergents et réémergents et aux menaces pour la santé publique» (9).

Un examen de la littérature de l'époque a cependant permis de trouver peu de références accessibles au public contenant des listes de contrôle concernant les interventions contre les maladies infectieuses émergentes et à conséquence grave. II est à noter que ces références, soit n'ont pas mis l'accent sur la composante laboratoire de la résilience de la santé publique, soit $n$ 'ont pas décrit les considérations d'intervention des laboratoires indépendamment des contextes organisationnels, juridictionnels ou spécifiques aux maladies infectieuses $(2,10,11)$. L'émergence récente de la nouvelle maladie infectieuse à coronavirus (maladie à coronavirus 2019, COVID-19), causée par le coronavirus du syndrome respiratoire aigu sévère 2 (SRAS-CoV-2), n'a fait que souligner l'importance cruciale d'une réponse rapide et coordonnée des laboratoires pour soutenir l'action de santé publique (12).

La liste de contrôle pour laboratoires d'intervention contre les éclosions de maladie infectieuse (la «liste de contrôle») a été élaborée pour fournir aux laboratoires un outil utile et adaptable leur permettant d'identifier rapidement les principales considérations relatives aux interventions en cas d'épidémie en utilisant une approche systématique, en particulier lorsqu'ils étudient une menace de maladie infectieuse jusqu'alors inconnue ou nouvellement définie. Afin d'inclure les considérations associées aux interventions prolongées des laboratoires à plus grande échelle, la liste de contrôle a été considérablement enrichie par les enseignements tirés des interventions antérieures du Laboratoire national de microbiologie contre le virus Ebola, le virus Zika et le syndrome respiratoire aigu sévère (SRAS) en 2003, et par la poursuite des efforts d'intervention contre la COVID-19 $(5-8,13,14)$. Comme les rôles d'intervention des laboratoires sont censés différer selon les organisations et les niveaux de juridiction, le contenu de la liste de contrôle n'a pas vocation à être prescriptif. La liste de contrôle a plutôt été conçue de manière à saisir l'étendue des considérations d'intervention qui peuvent être pertinentes à tous les niveaux, tout en soutenant la personnalisation du contenu afin de refléter les exigences spécifiques à chaque établissement. En tant que telle, la liste de contrôle est conçue comme un outil complémentaire aux plans et protocoles existants de préparation et d'intervention des laboratoires.

\section{Méthodes}

Un groupe de travail du Laboratoire national de microbiologie a été convoqué pour contribuer au développement de l'outil de la liste de contrôle afin de soutenir la coordination en temps utile des efforts d'intervention des laboratoires. Le groupe de travail était composé de plusieurs représentants de programmes ayant une expérience préalable dans l'exercice de fonctions au sein du centre des opérations d'urgence du Laboratoire national de microbiologie lors de l'activation de l'intervention contre les épidémies (15). Les membres du groupe de travail ont identifié les exigences et les défis rencontrés lors de I'intervention contre les éclosions de maladies infectieuses et aux menaces émergentes, tant au niveau national qu'international. Les informations recueillies ont été utilisées pour rédiger les considérations d'intervention afin d'obtenir une version préliminaire de la liste de contrôle. Afin de soutenir la mise en œuvre par les organisations de laboratoires opérant à différents niveaux de juridiction, l'élaboration de la liste de contrôle a été guidée par la nécessité d'intégrer les attributs clés suivants : adaptabilité, acceptabilité, évolutivité et facilité d'utilisation (16).

Pour améliorer l'adaptabilité de la liste de contrôle, les considérations d'intervention ont été décrites à un niveau élevé en utilisant, dans la mesure du possible, une terminologie générique et neutre par rapport au contexte. Cette approche a été adoptée pour permettre aux utilisateurs de modifier facilement le contenu en y incorporant la terminologie et les exigences opérationnelles préférées, propres à l'organisation et à la juridiction, élargissant ainsi la portée potentielle de la mise en place d'organisations et de réseaux de laboratoires aux niveaux régional, provincial, territorial, étatique, national 
et international. Le contenu des considérations d'intervention proposées a été évalué, et cinq thèmes d'intervention de laboratoire ont été identifiés pour faciliter leur utilisation. Le contenu de chaque thème a été conçu de manière à être évolutif, ce qui permet aux utilisateurs d'élargir ou de modifier la portée des points de la liste de contrôle en fonction des activités d'intervention pertinentes au rôle de l'organisation de laboratoires de mise en œuvre. Le tableau d'examen de la liste de contrôle qui en résulte (supplémentaire I) est conçu pour être reproductible et adaptable à l'aide de logiciels de tableur ou de base de données préférés, offrant la possibilité de sélectionner, de trier et de surveiller avec souplesse le statut des points marqués en fonction de l'utilisateur. Afin de faciliter son utilisation, un certain nombre d'approches permettant de classer, de personnaliser et de hiérarchiser les points de la liste de contrôle ont été élaborées pour être soumises à l'examen des utilisateurs (supplémentaire II).

L'ébauche de liste de contrôle a été pilotée à l'aide d'un exercice d'examen de documents basé sur un scénario auquel ont participé le personnel du centre des opérations d'urgence du Laboratoire national de microbiologie et le personnel de liaison des laboratoires. Les données de l'exercice ont été utilisées pour affiner le contenu et examiner la meilleure façon d'opérationnaliser la liste de contrôle afin d'améliorer les protocoles d'intervention d'urgence. Une boîte à outils supplémentaire d'intervention du Laboratoire national de microbiologie, qui contient les contacts, les références et les ressources des laboratoires spécifiques au contexte canadien, a été développée simultanément pour soutenir la mise en œuvre du Laboratoire national de microbiologie, et comme modèle adaptable par d'autres (disponible sur demande). Afin de vérifier l'acceptabilité et la validité du contenu, la liste de contrôle et la «trousse d'intervention du Laboratoire national de microbiologie» ont été distribuées pour examen à des experts de laboratoires fédéraux, provinciaux et territoriaux (Laboratoire national de microbiologie, Réseau des laboratoires de santé publique du Canada) et, au niveau international, au réseau de laboratoires du Groupe d'action pour la sécurité sanitaire mondiale. La liste de contrôle a été initialement distribuée sous deux formes : un «tableau d'examen de la liste de contrôle» triable (Microsoft Excel 2010) (supplémentaire I) et un document de «liste de contrôle » conventionnel formaté en « cases à cocher» (Microsoft Word 2010) (supplémentaire III). Les contributions des examens externes ont été prises en compte pour produire une version de référence finale de la liste de contrôle, qui a ensuite été distribuée aux parties prenantes du Laboratoire national de microbiologie, du Réseau des laboratoires de santé publique du Canada et du Réseau de laboratoires du Groupe de travail sur la protection de la santé mondiale en 2018. L'examen intergouvernemental a indiqué que la liste de contrôle était bien alignée sur les fonctions essentielles des laboratoires de santé publique déjà identifiées et sur les priorités définies dans le Plan stratégique 2016-2020 du Réseau des laboratoires de santé publique du Canada $(9,17,18)$. Le contenu a ensuite été mis à jour pour produire la version actuelle, qui inclut le contexte d'intervention des laboratoires contre la COVID-19.

\section{La liste de contrôle}

La liste de contrôle englobe cinq thèmes centraux : les études en laboratoire; la capacité d'intervention et la formation des laboratoires; la surveillance et la gestion des données des laboratoires; l'engagement et la communication entre les juridictions; et la recherche et l'éthique (supplémentaires I et III). Chaque thème comprend de multiples considérations identifiées comme pouvant avoir un impact sur l'intervention stratégique d'un laboratoire.

\section{1. Études en laboratoire}

Lors de la recherche d'un agent pathogène nouveau ou émergent, les considérations d'intervention rapide comprennent l'élaboration, la validation, le partage et la mise en œuvre de protocoles de test et de recommandations fondés sur des données probantes, en étroite collaboration avec les laboratoires partenaires interjuridictionnels. L'établissement de critères normalisés de confirmation des cas par les laboratoires peut également être considéré comme une priorité afin de favoriser une déclaration et une surveillance cohérente des cas dans les juridictions concernées (2). Le développement rapide des capacités de dépistage a été une considération essentielle dès le début de l'intervention nationale du Canada contre la COVID-19. En prévision de l'arrivée de la COVID-19 au Canada, le Laboratoire national de microbiologie a travaillé à la mise au point de méthodes de diagnostic moléculaire qui ont été utilisées pour confirmer avec succès le premier cas présumé positif de COVID-19 en janvier 2020. Le Laboratoire national de microbiologie a continué à fournir des tests confirmatoires de référence et un soutien en matière d'assurance qualité aux partenaires provinciaux et territoriaux des laboratoires de santé publique qui ont répondu, afin de garantir l'exactitude permanente de la détection des cas à l'échelle nationale (19).

Les efforts d'intervention peuvent également exiger un dépistage et/ou des tests de confirmation de première ligne soutenus et en grand volume, dépassant les capacités habituellement disponibles des laboratoires. Dans ces circonstances, la mise en œuvre de critères de tests et de protocoles de triage basés sur des facteurs de risque connus peut devenir une considération pour hiérarchiser les études de laboratoire de manière appropriée et pour gérer des ressources limitées. Il s'agissait là d'une considération essentielle du Laboratoire national de microbiologie lors de sa première intervention domestique contre l'épidémie de Zika, qui a posé d'importants défis de capacité, car la demande de tests de dépistage de Zika a persisté à des niveaux élevés bien au-delà de la période d'intervention initiale de 2015 à 2016 (6). Lors de la première intervention contre la COVID-19, les provinces et territoires canadiens ont également établi des critères de tests afin de prioriser les tests selon les capacités des laboratoires. 
Cette approche a été adoptée, car les demandes mondiales écrasantes sur les chaînes d'approvisionnement internationales ont eu un impact significatif sur la capacité d'obtenir rapidement les fournitures, l'équipements et les réactifs de laboratoire nécessaires, notamment l'équipement de protection individuelle, les écouvillons de prélèvement, les milieux de transport viraux, les trousses de test, les réactifs et les plateformes de test $(20,21)$.

La liste de contrôle identifie diverses considérations d'intervention susceptibles d'influencer la coordination en temps utile des études de laboratoire, allant des exigences en matière de prélèvement et de transport des échantillons, aux tests de laboratoire et à la communication des résultats. Ces considérations comprennent 1) la capacité à prélever, entreposer et transporter des échantillons pour répondre aux critères d'acceptation d'échantillons pour les tests, 2) les considérations de biosécurité, de sûreté biologique et de contrôle des infections, 3) les exigences législatives et réglementaires, 4) les tests de laboratoire et les critères de confirmation des cas et 5) les protocoles de triage pour les tests prioritaires. Le contrôle de la qualité, la normalisation des méthodes de test ainsi que les processus de communication des résultats ont été jugés essentiels à tous les aspects d'une intervention coordonnée des laboratoires.

\section{Capacité d'intervention des laboratoires et formation}

Pour les laboratoires de santé publique intervenants, une prise en compte précoce de la capacité peut être la disponibilité de méthodes validées de dépistage en première ligne et de tests de diagnostic de confirmation spécifiques aux agents pathogènes avec des caractéristiques de performance connues. On peut s'attendre à ce que les laboratoires nationaux de référence conservent la capacité de développer et de valider rapidement de nouvelles méthodes de test lorsque les capacités externes n'existent pas, ou ne sont pas disponibles de manière fiable dans des circonstances d'épidémie (2). Les laboratoires de santé publique peuvent également être tenus de participer à la recherche coordonnée et interjuridictionnelle de fournisseurs et à la validation clinique continue des méthodes, équipements et fournitures de laboratoire, en particulier lorsqu'ils ont affaire à des fournisseurs multiples et/ou changeants pour gérer les défis de continuité de la chaîne d'approvisionnement, comme lors de l'intervention contre la COVID-19 (21). Les exigences de cette nature, qui sont sensibles au facteur temps et exigent beaucoup de ressources, peuvent imposer des contraintes considérables au personnel scientifique et technique concerné, car il faut parallèlement maintenir les activités de programme habituelles et mandatées.

La capacité d'intervention des laboratoires peut être davantage compliquée lorsque le personnel est affecté par les mesures imposées par le gouvernement pour prévenir la transmission au niveau communautaire d'une menace de maladie infectieuse émergente ou par la contraction d'une maladie associée à une maladie infectieuse émergente. Comme on l'a vu lors de I'intervention contre la COVID-19, ces mesures peuvent inclure de longues périodes d'auto-isolement ou de quarantaine en raison d'une maladie confirmée, d'une maladie symptomatique ou d'une exposition potentielle (contact avec un cas, antécédents de voyage); ainsi que le «travail à distance» et les modalités de travail alternatives nécessaires pour gérer les problèmes de garde d'enfants et de famille résultant de la fermeture d'écoles et d'autres mesures de maintien à domicile et de distanciation physique $(22,23)$.

L'engagement et la mobilisation de personnel possédant les compétences et l'expérience essentielle en matière d'intervention peut jouer un rôle central pour relever les défis liés à la capacité de pointe, tant à l'intérieur qu'à l'extérieur d'une organisation de laboratoires d'intervention, comme l'a démontré le Laboratoire national de microbiologie lors des interventions nationales dans le cadre de la COVID-19 et du virus Zika, ainsi que le déploiement international d'équipes d'intervention de laboratoire mobile pour soutenir le dépistage sur site du virus Ebola durant l'épidémie d'Ebola de 2014 à 2016 en Afrique occidentale (5). Le Laboratoire national de microbiologie a également déployé un laboratoire mobile sur le territoire national afin d'aider à tester les voyageurs canadiens récemment retournés au pays sur des sites de quarantaine pendant la première intervention contre la pandémie de COVID-19.

En fonction de l'ampleur et de la durée des besoins d'intervention, la décentralisation des tests de diagnostic et d'autres activités de transfert de technologie peuvent également être privilégiées pour étendre les capacités des laboratoires interjuridictionnels sur une base temporaire ou à long terme, et pour améliorer l'accès au dépistage des populations éloignées ou isolées (24). Au cours des interventions nationales canadiennes contre le virus Ebola, le virus Zika et, plus récemment, la COVID-19, le Laboratoire national de microbiologie et ses homologues provinciaux des laboratoires de santé publique ont travaillé en collaboration pour soutenir la décentralisation des tests de laboratoire de première ligne dans certaines juridictions afin d'améliorer la répartition des capacités d'intervention dans la mesure du possible, tout en maintenant une capacité nationale centralisée pour les tests de référence et de confirmation $(21,25)$.

La capacité à relever avec souplesse les défis en matière de capacité et d'aptitude propres aux menaces nouvelles ou aux maladie infectieuse émergente a été jugée essentielle aux efforts d'intervention. Les considérations connexes comprennent l'identification collaborative des capacités d'intervention des laboratoires avec les partenaires de santé publique interjuridictionnels, et l'évaluation dynamique de la capacité de pointe et des besoins de formation pour soutenir les activités essentielles à l'intervention. 
La liste de contrôle met en évidence diverses considérations qui peuvent être explorées si une capacité de pointe supplémentaire est nécessaire. II s'agit notamment d'étudier des approches alternatives pour améliorer la capacité en tests de laboratoire et le partage des informations, d'engager le soutien du site du centre des opérations d'urgence pour la coordination des interventions et d'identifier le personnel de capacité de pointe ayant les compétences requises par le biais de processus d'inventaire du personnel axé sur les interventions. Le personnel de surtension peut compléter une formation polyvalente et être mobilisé à un site de laboratoire alterne au sein ou à l'extérieur de l'organisation, ou encore être déployé sur le terrain sous la supervision de personnel scientifique de haut niveau dans le cadre d'une équipe d'intervention de laboratoire mobile.

\section{Surveillance et gestion des données des laboratoires}

Les données de résultats de laboratoire sont bien connues comme un apport essentiel pour soutenir les études épidémiologiques, la surveillance et les actions de santé publique liées aux maladies infectieuses $(26,27)$. Les activités d'intervention du Laboratoire national de microbiologie ont souligné que les études de laboratoire dépendent souvent de la disponibilité en temps utile de données épidémiologiques pour éclairer les processus de triage des tests, la sélection d'algorithmes de diagnostic et de confirmation appropriés ainsi que l'interprétation appropriée des résultats des tests. Lors de l'intervention nationale contre le virus Zika, la capacité à trier et à acheminer les échantillons en utilisant des facteurs de risque connus reposait sur la fourniture de données épidémiologiques et cliniques dans le cadre du processus de demande de tests (e.g. état de grossesse, antécédents de voyage et date de début des cas symptomatiques). De même, le rapprochement entre les résultats des tests du Laboratoire national de microbiologie et les cas faisant l'objet d'une étude dépendait de la fourniture d'identificateurs uniques appropriés (28).

Pour favoriser une surveillance et une gestion des données en laboratoire intégrées et en temps utile, les considérations d'intervention rapide peuvent inclure l'élaboration rapide, itérative et consensuelle d'une définition de cas de maladie infectieuse qui intègre les critères de confirmation des cas en laboratoire et épidémiologiques pertinents dans le contexte de l'épidémie actuelle (24). L'identification des éléments de données nécessaires aux études, à la confirmation des cas et aux efforts de surveillance des laboratoires fait partie intégrante de ce processus. L'harmonisation des critères de confirmation et des exigences relatives aux éléments de données entre les juridictions rapportant les cas peut mériter d'être prise en considération pour assurer la cohérence de la détection, des rapports et de la surveillance des cas, ainsi que la comparabilité des données de surveillance infranationales, nationales et internationales dans la mesure du possible. Une considération liée à la surveillance est la capacité de relier rapidement les résultats de laboratoire aux cas faisant l'objet d'une étude, et de relier les cas confirmés à une éclosion ou à une source d'éclosion. Le rapprochement et l'intégration des données peuvent s'avérer difficiles lorsque les éléments de données de laboratoire et épidémiologiques pertinents pour les études sur les cas sont générés ou recueillis par des juridictions de santé publique distinctes.

Les activités de surveillance et d'intervention des laboratoires peuvent être encore renforcées par la mise en œuvre d'approches génomiques normalisées en matière de santé publique et d'autres outils avancés d'épidémiologie moléculaire afin d'améliorer la détection, la caractérisation, l'attribution de la source et l'identification du mode de transmission des agents pathogènes et des éclosions. L'utilisation de méthodes de séquençage du génome entier pour soutenir la surveillance en temps réel, en laboratoire, de certains agents pathogènes offre également l'avantage promis d'une nomenclature non ambiguë à des fins de comparaison entre juridictions (29).

La liste de contrôle présente un certain nombre de considérations qui peuvent avoir une incidence sur la surveillance et la gestion des données par les laboratoires. Un examen des exigences en matière de flux de données peut être utile dès le début de l'intervention contre une menace de maladie infectieuse émergente, notamment la nécessité d'adopter des approches normalisées pour documenter, surveiller et communiquer les résultats des études de laboratoire et les informations récapitulatives sur l'épidémie afin de répondre aux besoins de renseignements des diverses parties prenantes (e.g. rapports de cas confirmés en laboratoire par juridiction, pourcentage de tests positifs par rapport au total des tests effectués pour les groupes ou populations cibles). II peut également être utile d'explorer le potentiel des systèmes existants de gestion des informations de laboratoire, des plateformes informatiques et de surveillance de la santé publique basées sur le Web, et d'autres outils permettant de répondre avec souplesse aux exigences de surveillance et $d^{\prime}$ intervention spécifiques aux agents pathogènes. Comme on l'a observé lors de la participation du Laboratoire national de microbiologie aux premières activités d'intervention contre la COVID-19, les fonctions souhaitées peuvent comprendre la collecte, le rapprochement et l'intégration de données en temps utile et en toute sécurité; la communication des résultats des tests de laboratoire et des indicateurs de surveillance, l'alerte de santé publique et la modélisation prédictive (30-32). Pour les laboratoires partenaires qui mettent en œuvre des approches "omiques» afin d'améliorer les capacités de détection et d'intervention contre les épidémies, les considérations à plus long terme peuvent inclure les besoins opérationnels et d'infrastructure pour les pipelines de transfert de données et les outils bio-informatiques utilisés pour transmettre, acquérir, analyser, interpréter et rapporter les résultats du séquençage du génome entier et autres résultats «omiques» $(27,32,33)$. Le partage entre juridictions des données de surveillance générées par les laboratoires peut également être envisagé dans le contexte des cadres législatifs et réglementaires pertinents et 
des accords de partage d'informations, conformément aux rôles établis des juridictions et des organisations en matière de santé publique (33-37).

\section{Engagement et communication interjuridictionnels}

Pour soutenir l'élaboration, fondée sur des données probantes, de directives de laboratoire, de recommandations cliniques, de stratégies de surveillance et d'intervention en santé publique, il peut être important d'envisager le renforcement des mécanismes de partage de renseignements avec les partenaires de santé publique concernés et les réseaux interjuridictionnels $(14,38)$. Cela peut impliquer l'exploration d'autres approches collaboratives et pluridisciplinaires. Au Canada, le Réseau des laboratoires de santé publique du Canada complète une fonction essentielle en fournissant un réseau établi, soutenu par un secrétariat et des experts nationaux, provinciaux et territoriaux en matière de laboratoires de santé publique afin d'appuyer l'élaboration rapide, par consensus, de stratégies d'intervention, de recommandations et de lignes directrices (9).

Les efforts d'intervention du Laboratoire national de microbiologie ont également souligné l'importance d'identifier clairement les structures de communication et de rapport spécifiques à l'événement pour soutenir un acheminement interne et externe efficace des demandes et des informations. Il s'agit notamment d'une messagerie cohérente utilisant des points de contact à "guichet unique " pour les programmes et les groupes de travail qui répondent au sein de l'organisation de laboratoires chaque fois que cela est possible. De telles considérations ne sont pas propres à l'intervention des laboratoires; la nécessité de rôles de direction et de structures de communication clairement articulés a également été identifiée dans une évaluation des considérations plus larges de résilience de la santé publique liées à l'intervention contre la maladie du virus Ebola au niveau communautaire aux États-Unis (10). Pour faciliter l'acheminement des demandes externes urgentes, le Laboratoire national de microbiologie a réussi à fournir un accès à guichet unique au soutien des laboratoires via un numéro de contact d'urgence 24 heures sur 24 , combiné à un soutien du centre des opérations d'urgence basé sur site pour coordonner et diriger de manière centralisée les demandes dans le contexte d'un système de commandement d'incidents (15). Le Laboratoire national de microbiologie tient également à jour un guide des services de laboratoire, accessible au public via le Web, qui sert de référence pour les demandes de test externes (28).

L'augmentation de la fréquence des engagements interjuridictionnels et des exigences en matière de communication des risques, qui sont sensibles au facteur temps, peut poser des défis permanents à la coordination d'intervention, en particulier lorsqu'un événement de santé publique suscite une préoccupation du public, un intérêt médiatique et une attention politique importante durant une période prolongée. Les experts en laboratoire chargés de l'intervention organisationnelle peuvent également être ceux qui sont les plus sollicités pour répondre aux demandes d'informations provenant de sources multiples.

Comme indiqué dans la liste de contrôle, une méthode de communication stratégique peut devenir cruciale pour préserver le temps précieux du personnel d'intervention, faciliter l'engagement coordonné des parties prenantes et assurer la cohérence des messages de renseignements sur la santé publique afin de répondre aux besoins spécifiques du public. L'utilisation d'outils de médias sociaux et d'autres plateformes Web peut permettre d'améliorer l'accessibilité des conseils de laboratoire aux professionnels de la santé publique, aux médias et au public.

\section{Recherche et éthique}

Au Canada, l'engagement actif dans la recherche liée à la santé publique est considéré comme une fonction essentielle des laboratoires de santé publique, car le maintien continu de cette capacité scientifique fournit les bases nécessaires à une action de santé publique adaptée $(13,17)$. Comme cela a été démontré lors de l'intervention contre l'épidémie de coronavirus du SRAS en 2003, les priorités immédiates de la recherche en santé publique relatives aux laboratoires peuvent inclure l'identification et la caractérisation rapides des agents pathogènes, y compris le séquençage génomique (39). Lancée en réponse à la pandémie de COVID-19 de 2020, I'initiative du Réseau canadien de génomique COVID permettra, avec la participation des laboratoires de santé publique, de créer une base de données génomiques "du virus au patient» par le séquençage à grande échelle des génomes de l'hôte et du virus, afin de soutenir la recherche nationale et internationale sur la pathogénicité virale, les résultats en termes d'évolution et de santé, et les vaccins et thérapeutiques (40).

D'autres priorités d'intervention immédiate peuvent inclure l'élaboration et la validation de méthodes de tests diagnostiques, et la recherche appliquée en biosécurité avec un accent sur l'application rapide des connaissances. La transmission des agents pathogènes et les études sur la compétence des vecteurs peuvent également être prioritaires pour aider à caractériser les risques et à informer les stratégies de prévention. Tout comme durant l'intervention contre le virus Zika, cela peut être particulièrement pertinent lorsque le potentiel d'introduction et de transmission soutenue d'une maladie émergente à transmission vectorielle doit encore être évalué dans des contextes non endémiques (41).

Pour réduire la morbidité et la mortalité au sein des populations à risque, les priorités nationales de recherche peuvent s'étendre à une élaboration et une mise en œuvre concertées d'interventions de santé publique, y compris des vaccins et d'autres contre-mesures médicales, comme le montre I'intervention du Laboratoire national de microbiologie contre la maladie du virus Ebola $(42,43)$. La poursuite des efforts d'intervention contre la pandémie de COVID-19 a démontré la 
nécessité impérative de comprendre la réponse immunitaire de I'hôte à l'infection, ce qui permettra d'éclairer les stratégies de tests de laboratoire visant à déterminer le statut immunitaire au niveau des individus et de la population (e.g. par des études de séroprévalence), ainsi que les stratégies d'élaboration et de mise en œuvre de thérapies et de vaccins (44). En avril 2020, le gouvernement du Canada a lancé le groupe de travail sur I'immunité à la COVID-19, réunissant des experts nationaux du monde universitaire, hospitalier et de la santé publique pour aider à répondre aux questions en suspens liées à l'immunité à la COVID-19, notamment 1) le statut immunitaire et la durée de l'immunité après l'infection et 2) la portée de l'immunité au niveau de la population pour soutenir les efforts nationaux d'intervention contre les épidémies (45).

Face à une menace de maladie infectieuse émergente, il peut être urgent d'orienter stratégiquement les activités de recherche en santé publique afin de combler d'importantes lacunes dans les connaissances scientifiques et les capacités techniques. Les niveaux d'engagement dans la recherche peuvent varier considérablement d'un laboratoire à l'autre, en fonction du contexte juridictionnel et des responsabilités en matière de santé publique.

Comme indiqué dans la liste de contrôle, une première réaction peut consister à donner la priorité aux activités de recherche collaborative dans le cadre des ressources et des capacités existantes. Les priorités de recherche peuvent comprendre des études d'identification et de caractérisation des agents pathogènes, des études d'élaboration et de validation de méthodes de diagnostic et de tests de référence, des recherches sur l'immunité au niveau de l'hôte et de la population, des études sur la transmission et la compétence des vecteurs, l'élaboration et l'évaluation de vaccins et d'autres contremesures médicales, la recherche appliquée en matière de biosécurité et des études de surveillance de la santé publique. Pour rationaliser la coordination des interventions, il peut également être utile d'établir une distinction claire entre la recherche appliquée en santé publique et les activités de surveillance qui font partie intégrante des interventions de routine des laboratoires, et les autres activités de recherche ciblées qui nécessiteront un consentement préalable et l'achèvement des processus d'approbation éthique de la recherche.

Les groupes de travail et les réseaux d'intervention contre les maladie infectieuse émergente peuvent également être convoqués pour mener des initiatives de recherche hautement prioritaires et urgentes, en faisant appel à l'expertise des laboratoires de santé publique ainsi qu'à d'autres experts scientifiques des universités, des hôpitaux et du secteur privé. Lorsque la recherche collaborative implique des partenaires interjuridictionnels ou des équipes multidisciplinaires, il peut être nécessaire de coordonner les processus d'évaluation de plusieurs comités d'éthique de la recherche et d'aborder les questions liées à la paternité et à la propriété intellectuelle d'une manière qui favorise la publication en temps voulu pour éclairer la prise de décision en matière de santé publique.

\section{Discussion}

\section{Interdépendance des fonctions des laboratoires de santé publique}

Lors de l'évaluation et de la hiérarchisation des considérations relatives à la préparation et à l'intervention des laboratoires, il est important de noter qu'il existe des interdépendances importantes entre les fonctions clés des laboratoires de santé publique, qui comprennent les éléments suivants : tests de diagnostic et de référence; surveillance des maladies infectieuses; préparation et intervention en cas d'éclosion épidémique; et recherche fondamentale et appliquée (figure 1). À l'interface de ces fonctions cycliques et adjacentes des laboratoires de santé publique, les exigences d'intervention contre les maladie infectieuse émergente sont fortement interdépendantes. Une intervention de santé publique solide, capable de satisfaire à chacune de ces exigences mutuellement interdépendantes, dépend de la capacité disponible du système des laboratoires de santé publique à remplir chacune de ses fonctions essentielles.

\section{Figure 1 : Interdépendances des fonctions d'intervention des laboratoires de santé publique}

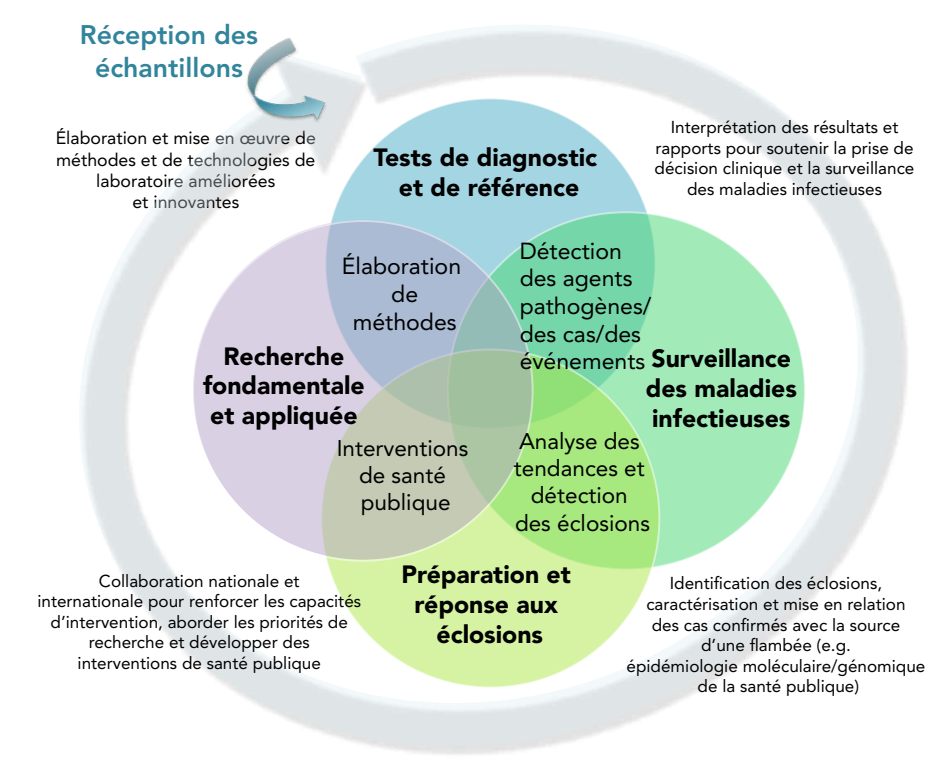

La figure 1 est un diagramme de Venn composé de quatre cercles qui se chevauchent, chaque cercle représentant une fonction distincte des laboratoires de santé publique, et chaque zone de chevauchement indiquant son interdépendance avec la fonction immédiatement adjacente. Le diagramme de Venn est circonscrit par une flèche circulaire, orientée dans le sens horaire, qui indique la nature cyclique et ordinale des quatre fonctions, en débutant avec la réception d'un échantillon à tester par le 
laboratoire, qui est représenté comme une entrée externe au diagramme de Venn. Les interdépendances indiquées par les domaines de chevauchement entre les quatre fonctions des laboratoires de santé publique comprennent les capacités des laboratoires pour les éléments suivants :

1. La détection des agents pathogènes, des cas et des événements, y compris l'interprétation et la communication des résultats des tests pour soutenir à la fois la prise de décision clinique et les activités de surveillance des maladies infectieuses

2. L'analyse des tendances et la détection des éclosions y compris les activités de détection et de surveillance des maladies pour permettre l'identification et la caractérisation des éclosions en temps utile et la mise en relation des cas confirmés avec la source des éclosions (e.g. à l'aide de méthodes d'épidémiologie moléculaire/génomique de la santé publique)

3. Les interventions de santé publique, y compris la collaboration nationale et internationale pour renforcer les capacités d'intervention et faire progresser la recherche sur les interventions de santé publique, notamment la mise au point de contre-mesures médicales telles que des vaccins et d'autres approches thérapeutiques

4. L'élaboration de méthodes, y compris l'élaboration et la mise en œuvre de méthodes et de technologies de laboratoire améliorées et innovantes

Dans le contexte des fonctions interdépendantes des laboratoires de santé publique, il devient évident que les considérations individuelles identifiées dans chacun des cinq thèmes d'intervention de la liste de contrôle peuvent avoir des implications importantes et de grande portée sur les activités d'intervention interdépendantes dans le contexte plus large de la santé publique. Par exemple, I'utilisation de méthodes d'investigation de laboratoire normalisées pour la détection des cas (Thème 1 : Études en laboratoire) peut faciliter des mécanismes de surveillance fiables et bien intégrés qui, à leur tour, permettent la détection et le suivi interjuridictionnels des cas et des éclosions en temps opportun (Thème 3 : Surveillance et gestion des données des laboratoires). Les renseignements sur la santé publique générés grâce à des activités de surveillance de haute qualité axées sur des objectifs et partagés en temps opportun via des réseaux d'experts collaboratifs (Thème 4 : Engagement et communication interjuridictionnels) peuvent alors permettre l'identification des priorités de recherche pour éclairer les efforts de préparation et d'intervention à court et à long terme (Thème 5 : Recherche et éthique). La mobilisation stratégique et la formation des postes pour la capacité de pointe peuvent être nécessaires afin de soutenir les activités prioritaires à travers le continuum d'intervention (Thème 2 : Capacité d'intervention et formation des laboratoires).

Lorsque I'on opère dans des environnements aux ressources limitées et sous des contraintes de temps importantes, les oublis lors de la phase initiale de planification peuvent avoir un impact sur l'efficacité globale de l'intervention de diverses manières. Les conséquences peuvent comprendre un manque d'allocation de ressources pour soutenir les priorités d'intervention des laboratoires qui ont été négligées, des retards ou des perturbations dans le partage de l'intelligence scientifique ou l'acquisition et la distribution de matériel et de produits biologiques, l'absence de représentation appropriée des laboratoires aux principales tables de décision et d'autres problèmes en aval. L'importance de mécanismes de financement rapides a été soulignée par l'Organisation mondiale de la santé à la suite de l'intervention contre le virus Ebola en Afrique de l'Ouest, car «les éclosions de maladie évoluent souvent plus vite que les fonds alloués pour y faire face» (46).

Un défi particulier peut être la capacité de maintenir les activités régulièrement mandatées en parallèle avec les demandes des efforts d'intervention des laboratoires en évolution, et peut nécessiter une évaluation minutieuse de la capacité d'augmentation des effectifs et de la hiérarchisation des activités. Par exemple, la réaffectation interne de personnel hautement qualifié des programmes existants pour répondre aux besoins immédiats d'intervention contre la crise peut créer d'importantes lacunes opérationnelles dans toute l'organisation qui doivent également être comblées pour maintenir la capacité d'intervention globale en matière de santé publique. À long terme, les lacunes en matière d'expertise peuvent avoir un impact cumulatif sur les capacités des laboratoires au cours d'efforts d'intervention multiples, séquentiels ou simultanés. II n'est pas facile de remédier à ces insuffisances de capacité en utilisant des approches à court terme face à une menace émergente. Cet effet a été observé par le Laboratoire national de microbiologie peu après le début des activités d'intervention contre la COVID-19, et a été une considération permanente associée aux interventions antérieures contre les maladies infectieuses étendues. Bien que le problème ait été identifié pour la première fois dans le rapport Naylor de 2003, qui présentait en détail les leçons tirées de l'intervention du Canada en matière de santé publique contre la pandémie de SRAS, le $D^{r}$ David Butler-Jones a de nouveau souligné, le 3 février 2020, les répercussions possibles des lacunes plus récentes, après 2014, en matière d'expertise et de ressources de santé publique sur la capacité globale d'intervention en santé publique au Canada, quelques jours seulement après que l'Organisation mondiale de la santé ait déclaré que l'épidémie de maladie à coronavirus 2019 (nCoV-2019) était une urgence de santé publique de portée internationale le 30 janvier $2020(13,47,48)$.

\section{Mise en œuvre de la liste de contrôle - considérations supplémentaires}

La nature multidimensionnelle et interdépendante des considérations relatives à l'intervention des laboratoires pose divers défis aux efforts de coordination à haut niveau. En l'absence d'une approche stratégique, il existe un risque réel que toutes les considérations pertinentes au contexte d'intervention ne soient pas identifiées en vue d'une action en temps utile. 
Dans le contexte canadien, comme dans d'autres pays, il existe de nombreux documents d'orientation qui fournissent des recommandations approfondies sur les mesures à prendre en fonction des agents pathogènes et des maladies. Celles-ci vont des méthodes d'essai, des procédures opérationnelles et des protocoles d'intervention en cas d'épidémie normalisés utilisés dans les environnements de laboratoire, aux directives de planification et d'intervention en cas d'urgence utilisées à la fois par les organisations et par les réseaux de laboratoires interjuridictionnels $(28,49-51)$. Les exigences législatives et réglementaires spécifiques à chaque juridiction définissent les paramètres dans lesquels les activités d'intervention des laboratoires sont menées pour soutenir la biosécurité, la sûreté biologique et la confidentialité des informations; tandis que d'autres réglementations et accords multilatéraux de partage d'informations (e.g. I'Entente multilatérale sur l'échange de renseignements) définissent des exigences et des principes spécifiques aux maladies permettant l'échange de renseignements entre juridictions afin de soutenir une surveillance et une intervention en temps utile en cas d'éclosion. $(34,35,52,53)$.

Sur le plan opérationnel, l'objectif de la liste de contrôle est de remplir une fonction complémentaire par rapport à d'autres documents d'orientation de laboratoire plus normatifs et spécifiques au contexte. La mise en œuvre d'une liste de contrôle souple et non prescriptive est proposée comme moyen de faciliter la coordination globale de l'intervention en identifiant et en hiérarchisant rapidement les considérations pertinentes à travers plusieurs thèmes d'intervention.

Bien que la liste de contrôle ait été conçue pour être facile à utiliser dans sa forme de référence actuelle, il est reconnu que toutes les considérations de la liste de contrôle peuvent ne pas être pertinentes ou ne pas entrer dans le cadre normal des activités d'intervention pour une entité de laboratoire donnée (e.g. recherche ciblée, élaboration de contre-mesures médicales). Ainsi, une personnalisation supplémentaire du contenu et de la terminologie de la liste de contrôle pour refléter les rôles et les responsabilités des laboratoires spécifiques au site peut optimiser l'utilité globale. La fonctionnalité peut être encore améliorée par l'élaboration d'annexes supplémentaires afin de saisir les références et les ressources importantes propres à chaque juridiction et afin d'aider à orienter les actions futures concernant toute considération identifiée comme pertinente au cours d'un processus d'examen donné de la liste de contrôle.

Les organismes et réseaux de mise en œuvre peuvent également souhaiter examiner les approches préférées pour faire participer les participants au processus d'examen de la liste de contrôle, y compris l'équilibre entre la représentation des experts en la matière et celle des travailleurs, nécessaire pour refléter la portée des activités d'intervention potentielles. Il peut être utile d'identifier les déclencheurs opérationnels susceptibles de démarrer un examen formel de la liste de contrôle, en reconnaissant que ces déclencheurs peuvent varier pendant et entre des circonstances d'éclosions, et peuvent être internes ou externes à l'organisation d'intervention. Par exemple, l'examen complet de la liste de contrôle peut être considéré comme une priorité organisationnelle immédiate lors d'une intervention contre des menaces de maladies infectieuses pour lesquelles des protocoles d'intervention doivent toujours être élaborés. L'examen peut également être lancé en réponse à un déclencheur externe pertinent, tel que l'identification d'une menace de maladie infectieuse émergente pouvant potentiellement avoir des conséquences graves, ou la déclaration officielle d'une urgence de santé publique de portée internationale par l'Organisation mondiale de la santé (36).

La mise en œuvre de la liste de contrôle peut être envisagée sur le plan opérationnel dans le contexte des outils de planification existants du programme de gestion des urgences afin d'améliorer les fonctions d'atténuation, de préparation, d'intervention et de rétablissement, conformément aux quatre phases de la gestion des urgences (15). Par exemple, un processus d'examen de la liste de contrôle peut être lancé dans le cas où les efforts d'intervention nécessitent un soutien d'urgence coordonné au niveau central, y compris l'activation officielle d'un centre des opérations d'urgence associé à l'organisation des laboratoires. L'examen par un groupe d'experts en la matière, représentatif des programmes engagés dans les activités d'intervention ou susceptibles d'être touchés par celles-ci, peut être lancé et coordonné par la direction des laboratoires ou par l'intermédiaire du centre des opérations d'urgence. Les considérations de haut niveau, les lacunes et les mesures proposées en rapport avec l'intervention actuelle peuvent être documentées à l'aide de la version électronique de l'outil de la liste de contrôle, puis distribuées pour le suivi, conformément au système de commandement des incidents actuellement en vigueur (15). Sur le plan opérationnel, un document de liste de contrôle permettant de contrôler la qualité et constamment mis à jour peut être maintenu de manière centralisée pour servir de référence à la planification pendant les périodes d'éclosion et entre ces périodes.

Si chaque processus d'examen de la liste de contrôle peut identifier de nombreuses considérations pertinentes, il peut être possible de n'agir que sur un sous-ensemble de considérations sensibles au temps au milieu d'une intervention, tout en reportant les autres pour une action future durant une période entre éclosions. II faut également considérer qu'une fois que l'intervention initiale contre une menace de maladie infectieuse émergente est terminée, les responsabilités des laboratoires mandatés peuvent ne pas revenir à leur niveau de référence d'avant l'éclosion, dans l'immédiat ou à long terme. À mesure que de nouveaux tests sont intégrés dans les menus des tests de laboratoire de routine et que les niveaux de tests restent élevés pour soutenir la détection et la surveillance des cas en cours, chaque intervention ultérieure contre la maladie infectieuse émergente peut avoir un impact cumulatif sur les 
activités de base des laboratoires, de sorte que des ressources supplémentaires sont nécessaires pour maintenir les activités entre les éclosions à des niveaux «nouvellement normaux». L'examen périodique des considérations de la liste de contrôle pendant les périodes entre deux épidémies peut aider à identifier les lacunes opérationnelles qui en résultent et à éclairer les efforts à plus long terme visant à renforcer les capacités d'intervention des laboratoires.

\section{Limites}

Le contenu de la liste de contrôle reflète principalement les perspectives et les expériences des laboratoires de santé publique intervenants canadiens et internationaux qui ont participé directement au processus d'élaboration et d'examen. Bien que les considérations relatives aux organisations de laboratoires opérant aux niveaux local et régional (e.g. les laboratoires hospitaliers et les laboratoires de diagnostic de première ligne) puissent différer en termes d'orientation et de portée, elles restent très pertinentes pour la réactivité globale de l'écosystème des laboratoires de santé publique. L'une des principales limites associées à l'initiative de la liste de contrôle est la mesure dans laquelle celle-ci sera mise en œuvre et utilisée comme prévu par les organisations et réseaux de laboratoires participant à l'intervention de santé publique.

\section{Conclusion}

Face à une menace de maladie infectieuse émergente, une intervention efficace des laboratoires nécessite la coordination, en temps utile, de multiples activités interdépendantes pour soutenir l'action de santé publique. Selon les premières informations recueillies, la liste de contrôle pourrait être un outil utile afin d'identifier rapidement et systématiquement les principales considérations en matière d'intervention, mettre en évidence les besoins et les lacunes opérationnels et éclairer la planification stratégique, l'établissement des priorités et la prise de décision visant à atténuer les risques.

L'un des principaux objectifs de l'initiative de la liste de contrôle était de garantir la disponibilité d'une version de référence pouvant être utilisée sous sa forme actuelle ou adaptée par les laboratoires la mettant en œuvre afin d'améliorer sa pertinence dans son cadre d'utilisation. Après sa mise en œuvre, la liste de contrôle est destinée à servir de "document vivant» pouvant être mis à jour pour refléter l'évolution des rôles, des considérations et des enseignements tirés des futurs efforts d'intervention des laboratoires.

La mise en œuvre, I'adaptation, l'examen et la mise à jour de routine de la liste de contrôle dans le contexte des cadres de gestion des urgences existants peuvent permettre de renforcer encore la préparation et les capacités d'intervention des laboratoires contre les éclosions, et de contribuer au développement de la résilience à long terme de la santé publique. À l'avenir, toute évaluation future de l'utilité de la liste de contrôle dans les organisations et les juridictions l'utilisant devra tenir compte de ces facteurs.

\section{Déclaration des auteurs}

Les contributions des auteurs sont les suivantes:

T. E. - Conceptualisation, développement du contenu (outil de la liste de contrôle, annexes, illustration des figures), rédaction, révision et édition

G. T. - Conceptualisation, révision et édition

T. K. - Conceptualisation, révision et édition

M. G. - Conceptualisation, révision et édition

\section{Intérêts concurrents}

Les auteurs n'ont pas d'intérêts concurrents.

\section{Remerciements}

Toutes les personnes et organisations et tous les réseaux ayant participé aux discussions techniques préliminaires, à l'élaboration et à la révision du document de la liste de contrôle sont remerciés pour leurs contributions, notamment :

Laboratoire national de microbiologie (Laboratoire national de microbiologie) : S. Beddome, Dr C. Corbett, K. Gordon, S. Guercio, C. Jorowski, L. Kearney, K. Keith, C. Ouellette, S. Radons Arneson, C. Yoshida, R. Cole (agent technique de liaison avec les laboratoires [ATLL], Manitoba [MB]), K. Cronin (ATLL, Ontario [ON]), R. DePaulo (ATLL, Saskatchewan [SK]), L. A. Jalbert (ATLL, Nouveau-Brunswick [NB]), K. Macdonald (ATLL, Colombie-Britannique [BC]), C. Phillips (ATLL, Nouvelle-Écosse [NS]), L. Wong (ATLL, Alberta $[A B])$

Réseau des laboratoires de santé publique du Canada (RLSPC) : Dr M. Gilmour (coprésident fédéral, Laboratoire national de microbiologie), Dr P. Van Caeseele (coprésident provincial, Cadham Provincial Laboratory, Winnipeg, MB), $D^{r}$ T. Kuschak (Laboratoire national de microbiologie, secrétariat du RLSPC), Dr M. Krajden (BC Centre for Disease Control Public Health Laboratory, Vancouver, BC), Y. Chang (BC Centre for Disease Control Public Health Laboratory, Vancouver, BC), Dr G. Tipples (Alberta Provincial Laboratory for Public Health, Alberta Precision Laboratories, Edmonton, AB), Dr P. Levett (Roy Romanow Provincial Laboratory, Regina, SK), Dr F. Jamieson (Public Health Ontario Laboratories, Toronto, ON), Dr J. Longtin (Laboratoire de santé publique du Québec, Ste-Anne-de-Bellevue, Québec [QC]), Dr G. German (Health Prince Edward Island [PEI], Charlottetown, PEI), Dr G. D. Haldane (Queen Elizabeth II Health Science Centre, Halifax, NS), $D^{r}$ G. Zahariadis (Newfoundland and Labrador [NL] Public Health Laboratory, St. John's, NL), Dr R. Garceau (Centre hospitalier universitaire $\mathrm{D}^{r}$ Georges-L.-Dumont, Moncton, NB)

Réseau de laboratoires du Groupe de travail sur la protection de la santé mondiale (GTPSM) : $\mathrm{D}^{r} \mathrm{M}$. Gilmour (coprésident, Winnipeg, MB, Canada), Dr J. A. Diaz Quiñonez (coprésident, Mexico City, Mexico), Dr T. Kuschak (chef du secrétariat, 
Winnipeg, MB, Canada), Dr A. Nitsche (Berlin, Allemagne), A. Roberts (Londres, Royaume-Uni), Dr A. Di Caro (Rome, Italie), C. Chadwick (Washington DC, États-Unis), Dr M. Saijo (Tokyo, Japon), Dr M. W. Shaw (Atlanta, États-Unis)

\section{Financement}

Ce travail a été soutenu par l'Agence de santé publique du Canada.

\section{Matériel supplémentaire}

Des documents supplémentaires pour cet article sont disponibles en ligne.

Supplémentaire I : Liste de contrôle pour laboratoires d'intervention contre les éclosions de maladie infectieuse (https://www.canada.ca/content/dam/phac-aspc/documents/ services/reports-publications/canada-communicable-diseasereport-ccdr/monthly-issue/2020-46/issue-10-oct-1-2020/ ccdrv46i10a01fs1s2-fra.xlsx)

Supplémentaire II : Liste de contrôle pour laboratoires d'intervention contre les éclosions de maladie infectieuse recommandations d'utilisation (https://www.canada.ca/content/ $\mathrm{dam} / \mathrm{phac}-\mathrm{aspc} / \mathrm{documents/services/reports-publications/canada-}$ communicable-disease-report-ccdr/monthly-issue/2020-46/issue10-oct-1-2020/ccdrv46i10a01fs1s2-fra.xlsx)

Supplémentaire III: Supplémentaire III : Liste de contrôle pour laboratoires d'intervention contre les éclosions de maladie infectieuse (https://www.canada.ca/content/dam/phac-aspc/ documents/services/reports-publications/canada-communicabledisease-report-ccdr/monthly-issue/2020-46/issue-10-oct-1-2020/ ccdrv46i10a01fs3-fra.pdf)

\section{Références}

1. Hitchcock P, Chamberlain A, Van Wagoner M, Inglesby TV, O'Toole T. Challenges to global surveillance and response to infectious disease outbreaks of international importance. Biosecur Bioterror 2007 Sep;5(3):206-27. DOI PubMed

2. Agence de la santé publique du Canada. Lignes directrices à l'intention des laboratoires : Préparation du Canada en cas de grippe pandémique : Guide de planification pour le secteur de la santé. Ottawa (ON) : ASPC; 2015. https:// www.canada.ca/fr/sante-publique/services/grippe-influenza/ preparation-canada-cas-grippe-pandemique-guide-planifica tion-secteur-sante/annexe-a-intention-des-laboratoires.html

3. Gardy JL, Loman NJ. Towards a genomics-informed, real-time, global pathogen surveillance system. Nat Rev Genet 2018 Jan;19(1):9-20. DOI PubMed
4. Yachison CA, Yoshida C, Robertson J, Nash JH, Kruczkiewicz P, Taboada EN, Walker M, Reimer A, Christianson S, Nichani A, Nadon C; PulseNet Canada Steering Committee. PulseNet Canada Steering Committee, Celine Nadon. The validation and implications of using whole genome sequencing as a replacement for traditional serotyping for a national Salmonella reference laboratory. Front Microbiol 2017 Jun;8:1044. DOI PubMed

5. Semalulu T, Wong G, Kobinger G, Huston P. Pourquoi l'éclosion de la maladie à virus Ebola est-elle si difficile à endiguer? Relevé des maladies transmissibles au Canada 2014;40(14):290-30. DOI

6. Agence de la santé publique du Canada. Recommandations sur la prévention et le traitement du virus Zika: Une déclaration du Comité consultatif, Comité consultatif de la médecine tropicale et de la médecine des voyages. Ottawa (ON) : ASPC; 2018 (accédé 2018-09-06). https:// www.canada.ca/fr/sante-publique/services/publications/ maladies-affections/recommandations-preventio n-traitement-virus-zika.html

7. Centers for Disease Control and Prevention. 2014 - 2016 Ebola outbreak in West Africa. CDC; 2017 (accédé 201809-06). https://www.cdc.gov/vhf/ebola/history/2014-2016outbreak/index.html

8. Pan American Health Organization. Regional Zika epidemiological update (Americas) August 25, 2017. Washington (DC): PAHO; 2017 (accédé 2018-09-06). https:// www.paho.org/hq/index.php?option=com_content\&view =article\&id=11599:regional-zika-epidemiological-updateamericas\&ltemid=41691\&lang=en

9. Réseau des laboratoires de santé publique du Canada. Plan stratégique 2016-2020 du Réseau des laboratoires de santé publique du Canada. RLSPC; 2018. https://www. canada.ca/fr/services/sante/publications/science-recherch e-et-donnees/2016-2020-plan-stratrgique-reseau-laboratoir es-sante-publique-canada.html

10. Sell TK, Shearer MP, Meyer D, Chandler H, Schoch-Spana M, Thomas E, Rose DA, Carbone EG, Toner E. Public Health Resilience Checklist for High-Consequence Infectious Diseases-Informed by the Domestic Ebola Response in the United States. J Public Health Manag Pract 2018 Nov/Dec;24(6):510-8. DOI PubMed

11. Association of Public Health Laboratories. Emerging Infectious Diseases Planning and Response Framework. April 2005:1-8. https://www.aphl.org/programs/infectious_ disease/Documents/EID_2005April_Emerging-Infectiou s-Diseases-Planning-and-Response-Framework.pdf

12. World Health Organization. Laboratory Testing for 2019 novel coronavirus (2019-nCoV) in suspected human cases. WHO; March 19, 2020 (accédé 2020-01-17). https:// www.who.int/publications-detail/laboratory-testing-for2019-novel-coronavirus-in-suspected-human-cases-20200117 
13. Santé Canada. Leçons de la crise du SARS : Renouvellement de la santé publique au Canada: Un rapport du Comité consultatif national sur le SRAS et la Santé publique Octobre 2003. Ottawa (ON) : SC; 2003. https://www. canada.ca/content/dam/phac-aspc/documents/services/ reports-publications/learning-sars-renewal-publi c-health-canada/lecons-crise-sras-f.pdf

14. Gouvernement du Canada. Maladie à coronavirus (COVID-19) : Mise à jour sur l'éclosion. Ottawa (ON) : Gouvernement du Canada (modifié 2020-09-04). https://www.canada.ca/en/public-health/services/ diseases/2019-novel-coronavirus-infection.html

15. Marcino D, Gordon K. Aperçu du programme de gestion des urgences du Laboratoire national de microbiologie. Relevé des maladies transmissibles au Canada. 2018;44(5):113-7. DOI

16. Gagliardi AR, Marshall C, Huckson S, James R, Moore V. Developing a checklist for guideline implementation planning: review and synthesis of guideline development and implementation advice. Implement Sci 2015 Feb;10(19):19. DOI PubMed

17. Réseau des laboratoires de santé publique du Canada. Core Functions of Canadian Public Health Laboratories. Winnipeg (MB): CPHLN; February 9, 2011 (accédé 2020-06-16). https://nccid.ca/wp-content/uploads/sites/2/2020/03/ CPHLN-Core-Functions.pdf

18. Association of Public Health Laboratories. The Core Functions of Public Health Laboratories. Silver Spring (MD): APHL (modifié 2014). https://www.aphl.org/aboutAPHL/ publications/Documents/APHLCoreFunctionsandCapabilitie s_2014.pdf

19. Gouvernement du Canada. COVID-19 : Mise à l'épreuve du Laboratoire national de microbiologie (modifié 2020-08-06). https://www.ic.gc.ca/eic/site/063.nsf/fra/98099.html

20. Crowe K. Why isn't Canada testing everyone for coronavirus? CBC News; posted Apr 09, 2020 (modifié 202004-13). https://www.cbc.ca/news/health/coronavirus-covid1 9-testing-canada-1.5527219

21. Pabbaraju K, Wong AA, Douesnard M, Ma R, Gill K, Dieu P, Fonseca K, Zelyas N, Tipples GA. A Public Health Laboratory Response to the Pandemic. J Clin Microbiol 2020 Jul;58(8):e01110-20. DOI PubMed

22. Gouvernement du Canada. Mesures communautaires de santé publique pour atténuer la propagation des maladies à coronavirus (COVID-19) au Canada. Gouvernement du Canada (modifié 2020-05-30; accédé 2020-06-11). https:// www.canada.ca/fr/sante-publique/services/maladies/201 9-nouveau-coronavirus/professionnels-sante/mesures-sant e-publique-utilisees-reduire-covid-19.html

23. Hirschfield K. Coronavirus: Two employees at National Microbiology Laboratory in Winnipeg test positive for COVID-19. Global News; posted April 9, 2020 (modifié 2020-04-10). https://globalnews.ca/news/6803268/ coronavirus-national-microbiology-lab-winnipeg/
24. Broadhurst MJ, Brooks TJ, Pollock NR. Diagnosis of Ebola Virus Disease: Past, Present, and Future. Clin Microbiol Rev 2016 Oct;29(4):773-93. DOI PubMed

25. The Canadian Press. Coronavirus: Canadian health authorities prepare to increase COVID-19 lab testing. Global News; posted March 5, 2020 (modifié 2020-03-05). https://globalnews.ca/news/6633801/coronavirus-canadia n-testing-labs/

26. Davis JR, Lederberg J, editors. Public Health Systems and Emerging Infections: Assessing the Capabilities of the Public and Private Sectors: Workshop Summary (2000). Chapter 2: Epidemiological Investigation. Institute of Medicine (US) Forum on Emerging Infections; Washington (DC): National Academies Press (US); 2000. https://www.ncbi.nlm.nih.gov/ books/NBK100248/

27. Simonsen L, Gog JR, Olson D, Viboud C. Infectious Disease Surveillance in the Big Data Era: Towards Faster and Locally Relevant Systems. J Infect Dis 2016 Dec;214 suppl_4:S380-5. DOI PubMed

28. Réseau canadien de renseignements sur la santé publique. Détection moléculaire du virus Zika par la méthode RT-PCR. Winnipeg (MB) : RCRSP; mai 2018. https://cnphi.canada.ca/ gts/reference-diagnostic-test/10983?labld=1022

29. Nadon C, Van Walle I, Gerner-Smidt P, Campos J, Chinen I, Concepcion-Acevedo J, Gilpin B, Smith AM, Man Kam K, Perez E, Trees E, Kubota K, Takkinen J, Nielsen EM, Carleton H; FWD-NEXT Expert Panel. PulseNet International: vision for the implementation of whole genome sequencing (WGS) for global food-borne disease surveillance. Euro Surveill 2017 Jun;22(23):30544. DOI PubMed

30. World Health Organization. Public health surveillance for COVID-19: Interim guidance. WHO; Aug 7, 2020. https://www.who.int/publications/i/item/who-2019-nCoV-su rveillanceguidance-2020.7

31. Henry B; Groupe de travail sur la préparation du Canada en cas de grippe pandémique. Préparation du Canada en cas de grippe pandémique: Stratégie de surveillance. Relevé des maladies transmissibles au Canada 2018;44(1):16-20. $\mathrm{DOI}$

32. Mukhi S, Dhiravani K, Micholson B, Yan L, Hatchard J, Mubareka S, Bergeron C, Beattie T. An innovative mobile data collection technology for public health in a field setting. Online J Public Health Inform 2018 Sep; 10(2):e202. DOI PubMed

33. The Integrated Rapid Infectious Disease Analysis (IRIDA) Platform. https://www.irida.ca/

34. PulseNet International. PulseNet Canada. (modifié 2019-0830; accédé 2020-06-11). https://pulsenetinternational.org/ networks/canada.asp 
35. Commissariat à la protection de la vie privée du Canada. Lois sur la protection des renseignements personnels au Canada. CVP (modifié 2019-09-16; accédé 2020-07-02). https://www.priv.gc.ca/fr/sujets-lies-a-la-protection-d e-la-vie-privee/lois-sur-la-protection-des-renseignementspersonnels-au-canada/

36. Organisation mondiale de la Santé. Règlement sanitaire international (2005) Troisième édition. OMS; 2016. p. 1-89. (accédé 2019-07-02). https://apps.who.int/iris/bitstream/han dle/10665/246187/9789242580495-fre.pdf?sequence=1 /

37. Réseau pancanadien de santé publique. Entente multilatérale sur l'échange de renseignements (EMER). RPSP : 2014 (accédé 2019-09-02). http://www.phn-rsp.ca/ pubs/mlisa-emer-fra.php

38. Global Health Security Initiative. Fifteenth Ministerial Meeting of the Global Health Security Initiative (GHSI): 11 December, 2014 - Tokyo, Japan. (accédé 2019-11-19). http:// ghsi.ca/ministerial-statements/tokyo-december-2014/

39. Marra MA, Jones SJ, Astell CR, Holt RA, Brooks-Wilson A, Butterfield YS, Khattra J, Asano JK, Barber SA, Chan SY, Cloutier A, Coughlin SM, Freeman D, Girn N, Griffith OL, Leach SR, Mayo M, McDonald H, Montgomery SB, Pandoh PK, Petrescu AS, Robertson AG, Schein JE, Siddiqui A, Smailus DE, Stott JM, Yang GS, Plummer F, Andonov A, Artsob H, Bastien N, Bernard K, Booth TF, Bowness D, Czub M, Drebot M, Fernando L, Flick R, Garbutt M, Gray M, Grolla A, Jones S, Feldmann H, Meyers A, Kabani A, Li Y, Normand S, Stroher U, Tipples GA, Tyler S, Vogrig R, Ward D, Watson B, Brunham RC, Krajden M, Petric M, Skowronski DM, Upton C, Roper RL. The Genome sequence of the SARS-associated coronavirus. Science 2003 May;300(5624):1399-404. DOI PubMed

40. Génome Canada. Génome Canada mène une initiative de génomique de 40émillions de dollars pour lutter contre la pandémie de COVID-19. Ottawa (ON) : Génome Canada (accédé 2020-06-11). https://www.genomecanada.ca/ en/news/genome-canada-leads-40-million-genomics-init iative-address-covid-19-pandemic

41. Dibernardo A, Turell MJ, Lindsay LR, Loomer C, Iranpour M. Vector competence of some mosquito species from Canada for Zika virus. J Am Mosq Control Assoc 2017 Dec;33(4):276-81. DOI PubMed

42. Wu S, Kroeker A, Wong G, He S, Hou L, Audet J, Wei H, Zhang Z, Fernando L, Soule G, Tran K, Bi S, Zhu T, Yu $X$, Chen W, Qiu X. An Adenovirus vaccine expressing Ebola virus variant Makona glycoprotein is efficacious in guinea pigs and nonhuman primates. J Infect 2017 Jan;215(1):S326-S332. DOI

43. Qiu X, Wong G, Audet J, Bello A, Fernando L, Alimonti JB, Fausther-Bovendo $H$, Wei $H$, Aviles J, Hiatt E, Johnson A, Morton J, Swope K, Bohorov O, Bohorova N, Goodman C, Kim D, Pauly MH, Velasco J, Pettitt J, Olinger GG, Whaley K, Xu B, Strong JE, Zeitlin L, Kobinger GP. Reversion of advanced Ebola virus disease in nonhuman primates with ZMapp. Nature 2014 Oct;514(7520):47-53. DOI PubMed
44. Tay MZ, Poh CM, Rénia L, MacAry PA, Ng LF. The trinity of COVID-19: immunity, inflammation and intervention. Nat Rev Immunol 2020 Jun;20(6):363-74. DOI PubMed

45. COVID-19 Immunity Task Force. The COVID-19 Immunity Task Force: Helping to Guide Canada's Epidemic Response. April 23, 2020 (accédé 2020-06-11). https://www. covid19immunitytaskforce.ca

46. World Health Organization. Ebola then and now: Eight lessons from West Africa that were applied in the Democratic Republic of Congo. WHO (modifié 2020-04). https://www.who.int/news-room/feature-stories/detail/ ebola-then-and-now

47. Butler-Jones D. A forgotten lesson of SARS: The need for public health specialists and expertise. The Globe and Mail; February 3, 2020 (modifié 2020-03-11). https://www. theglobeandmail.com/opinion/article-a-forgotten-lesso n-of-sars-the-need-for-public-health-specialists-and/

48. Organisation mondiale de la Santé. La flambée de 2019nCoV est une urgence de santé publique de portée internationale. OMS; le 31 janvier 2020. https://www. euro.who.int/fr/health-topics/health-emergencies/ international-health-regulations/news/news/2020/2/201 9-ncov-outbreak-is-an-emergency-of-international-concern

49. Agence de la santé publique du Canada. Modalités canadiennes d'intervention lors de toxi-infection d'origine alimentaire (MITIOA) : Guide d'intervention en cas d'éclosion multijuridictionnelle de maladie entérique. Ottawa (ON) : ASPC; 2017. https://www.canada.ca/fr/ sante-publique/services/publications/securite-et-risque-poursante/modalites-canadiennes-intervention-lorstoxi-infection-origine-alimentaire-mitioa-guide-interventi on-cas-eclosion-multijuridictionnelle-maladie-enterique. html\#prea

50. Agence de la santé publique du Canada. Lignes directrices à l'intention des laboratoires: Préparation du Canada en cas de grippe pandémique : Guide de planification pour le secteur de la santé. Ottawa (ON) : ASPC (modifié 2017-12-05). https://www.canada.ca/fr/sante-publique/ services/grippe-influenza/preparation-canada-cas-gripp e-pandemique-guide-planification-secteur-sante/ annexe-a-intention-des-laboratoires.html\#a13

51. Sécurité publique Canada. Plan nord-américain contre I'influenza animale et la pandémie d'influenza. SP. https:// www.securitepublique.gc.ca/cnt/rsrcs/pblctns/nml-pndmcnflnz/index-fr.aspx

52. Gouvernement du Canada. Loi sur les agents pathogènes humains et les toxines (L.C. 2009, ch. 24). Site Web de législation (Justice). Sanctionnée le 2009-06-23 https://laws. justice.gc.ca/fra/LoisAnnuelles/2009_24/

53. Gouvernement du Canada. Règlement sur les agents pathogènes humains et les toxines (DORS/2015-44). Site Web de législation (Justice). Règlement à jour 2020-09-22; dernière modification 2015-12-01. https://laws.justice.gc.ca/ fra/reglements/DORS-2015-44/ 